\title{
Darstellung des Zusammenhangs von Produktarchitektur- und Produktionssystemgestaltung in SysML
}

\author{
Representation of the connection between product architecture design and production \\ system design in SysML
}

\author{
Lea-Nadine Schwede ${ }^{1 *}$, Michael Winter ${ }^{2}$, Hermann Lödding ${ }^{2}$, Dieter Krause ${ }^{1}$ \\ ${ }^{1}$ Institute of Product Development and Mechanical Engineering Design, Hamburg University of Technology \\ 2 Institute of Production Management and Technology, Hamburg University of Technology \\ ${ }^{*}$ Korrespondierender Autor: \\ Lea-Nadine Schwede \\ Technische Universität Hamburg \\ Institut für Produktentwicklung und Konstruktionstechnik \\ Denickestraße 17 (L) \\ 21073 Hamburg Telefon: +49 40428782949 \\ Mail: lea.schwede@tuhh.de
}

\begin{abstract}
The product standardization favors the design of production systems in terms of automation. This is contrary to the strategy of product differentiation, which serves external market variety. The goal of this paper is the design of an overall system that leverages product differentiation while not derogating the production efficiency. For this purpose, module drivers are recorded in a literature research and examined with regard to their relevance for production. In a next step, the module drivers are linked to the impact model of modular product structures in a SysML-model, which is then examined with regard to indirect connections.
\end{abstract}

\section{Keywords}

Product Architecture, Production Systems, MBSE 


\section{Einleitung}

Die Produktentwicklung steht vor der Herausforderung, eine vom Markt geforderte und immer größer werdende externe Vielfalt abzudecken [1]. Gegenläufig bietet eine hohe Produktstandardisierung in der Produktion das Potenzial, mit stärker automatisierten Produktionssystemen eine höhere Produktivität und kürzerer Durchlaufzeiten zu erreichen.

Die modulare Produktarchitekturgestaltung stellt eine erste Herangehensweise aus Sicht der Produktentwicklung zur Bewältigung dieses Zielkonfliktes dar. Durch die Modularisierung von Produktarchitekturen soll die interne Vielfalt bei gleichbleibender externer Vielfalt reduziert werden [1]. In der modularen Produktarchitekturgestaltung sind Methoden für die Modularisierung etabliert, die Module ausgehend von sogenannten Modultreibern bilden. Zudem wurden generelle Auswirkungen von modularen Produktarchitekturen auf die Beschaffung und die Produktion bereits in der Literatur in einem Wirkmodell modularer Produktstrukturen dokumentiert [2]. In der Gestaltung von Produktionssystemen wird bei der Wahl des Fertigungsprinzips und der Materialflussart die jeweilige Produktarchitektur berücksichtigt.

Eine systematische, detaillierte Betrachtung der Schnittstellen erfolgte bisher nicht. So ist beispielsweise unbekannt, wie eine konkrete Veränderung der internen Vielfalt die logistischen Zielgrößen quantitativ beeinflusst. In der Praxis ist der Zusammenhang der internen Vielfalt und der logistischen Zielgrößen mit großer Wahrscheinlichkeit nicht linear und durch Sprünge geprägt. Daher soll zwischen Veränderungen der internen Vielfalt mit einer Auswirkung auf die logistischen Zielgrößen und einer Veränderung der internen Vielfalt ohne bedeutende Auswirkung auf die logistischen Zielgrößen unterschieden werden. Dieses Vorgehen setzt eine genaue Kenntnis des Verhaltens voraus. Durch eine beidseitige Betrachtung können die Potenziale, die die jeweiligen Strategien der Produktentwicklung und der Produktionsgestaltung bieten, abgestimmt und dadurch besser ausgeschöpft werden. Die Modellierung des Zusammenhangs mithilfe eines Model-based Systems Engineering (MBSE) Ansatzes verspricht eine Durchgängigkeit, die auch indirekte Zusammenhänge zwischen den Disziplinen aufdecken kann. Langfristig soll mit der durchgängigen Modellierung das Ziel verfolgt werden, eine abgestimmte Gestaltung der Produktarchitektur und des Produktionssystems zu ermöglichen. Die Modellierung in SysML ermöglicht eine standartisierte und transparente Kenntnis der Wechselwirkungen und der Einflussgrößen, wodurch diese frühzeitig in die Produktentwicklung bzw. in die Produktionsgestaltung integriert werden können. Das Ziel ist eine abgestimmte Gestaltung des Gesamtsystems, um differenzierte Produkte und effiziente Produktionssysteme zu ermöglichen. Die Positionierung zwischen einer hohen externen Variantenvielfalt zur Befriedigung der Kundenwüsche und ihren Kostennachteilen im Produktentstehungsprozess soll durch eine durchgängige und von beiden Fachbereichen lesbare Wissensbasis vereinfacht werden.

In diesem Paper liegt der Fokus auf der initialen Entwicklung eines erweiterbaren Modells, das erste Zusammenhänge der Disziplinen für die Entwicklung modularer Produktarchitekturen nutzbar macht. Dazu wird zunächst in Kapitel 2 der Stand der Forschung zu den zuvor angesprochenen Themenbereichen zusammengefasst und anschließend der Forschungsbedarf abgeleitet. Das Forschungsvorgehen wird in Kapitel 3 vorgestellt. In Kapitel 4 werden Ausschnitte des entwickelten Datenmodells vorgestellt und hinsichtlich der Datendurchgängigkeit untersucht. Abschließend folgen in Kapitel 5 eine Diskussion der Inhalte und ein Ausblick. 


\section{Stand der Forschung}

Die folgenden drei Abschnitte führen in den aktuellen Forschungsstand ein. Abschließend wird in Abschnitt 2.4 der Forschungsbedarf abgeleitet.

\subsection{Methodische modulare Produktarchitekturgestaltung}

Um Produktarchitekturen modular zu gestalten, sind in der Literatur unterschiedliche Modularisierungsmethoden zu finden [3]. Diese verhelfen dazu, eine hohe vom Kunden gewünschte externe Vielfalt bei geringer interner Vielfalt zu erreichen. Modularisierungsmethoden sind dabei grundsätzlich gleich aufgebaut. Nachdem die bestehenden Produktstrukturen in Komponenten zerlegt werden, werden diese in einem nächsten Schritt analysiert. Aufbauend auf der Analyse erfolgt die Reintegration der Komponenten in Module. Die Reintegration zu Modulen stellen dabei modulbildene Methodenschritte dar. Diese können unterteilt werden in technisch-funktionale und produktstrategische Methodenschritte. Ein Beispiel dafür stellt die Anwendung der Heuristiken nach Stone in Schritt 3 seiner Methode "Towards a Theory of Modular Design“ dar [4]. Die Grundlage dafür bietet eine umsatzorientierte Funktionsstruktur des zu modularisierenden Produkts. Module werden anschließend so gebildet, dass die Komponenten, welche zusammen eine Funktion erfüllen sollen, zu Modulen zusammengefasst werden. Viele Methoden, welche produktstrategische Methodenschritte enthalten, arbeiten hingegen mit sogenannten Modultreibern. Dieses Konzept hat Erixon in seiner Methode „Modular Function Deployment - A Method for Product Modularisation" etabliert [5]. Modultreiber sind dabei Aspekte aus den unterschiedlichen Phasen des Produktlebenszyklus, wie z.B. der Produktion, und stellen jeweils Gründe für die Modularisierung dar, wobei unter dem Begriff Modul nicht zwingend physische Module gemeint sind [6].

\subsection{Wirkmodell modularer Produktstrukturen}

Durch die Modularisierung von Produktstrukturen von Produktfamilien kann der Zielkonflikt zwischen der Bereitstellung einer großen externen Vielfalt und einer Minimierung der internen Vielfalt gelöst werden. Die Modularität stellt dabei eine graduelle Eigenschaft von Produktstrukturen dar. Durch die Stärkung unterschiedlicher Modularisierungseigenschaften und -merkmale können verschiedene modulare Produktstrukturen generiert werden. Die Entscheidung für die Ausprägung der Modularität ist dabei gerade in der frühen Produktentstehungsphase wichtig [7]. Durch die Kenntnis der unterschiedlichen Auswirkungen der Modularisierungseigenschaften und -merkmale kann diese Entscheidung unterstützt werden. Mögliche Auswirkungen modularer Produktstrukturen sind unter anderem in Anwendungsbeispielen von Modularisierungsmethoden oder in bereits bestehenden Wirkmodellen zu finden (z.B. [8], [9], [10]). Die bekannten Auswirkungen wurden in einer umfangreichen Literaturrecherche aufgenommen [11] und den Modularisierungseigenschaften und -merkmalen in einem Wirkmodell modularer Produktstrukturen zugeordnet [2]. Ein Ausschnitt des Wirkmodells modularer Produktstrukturen ist in Bild 1 zu sehen. 


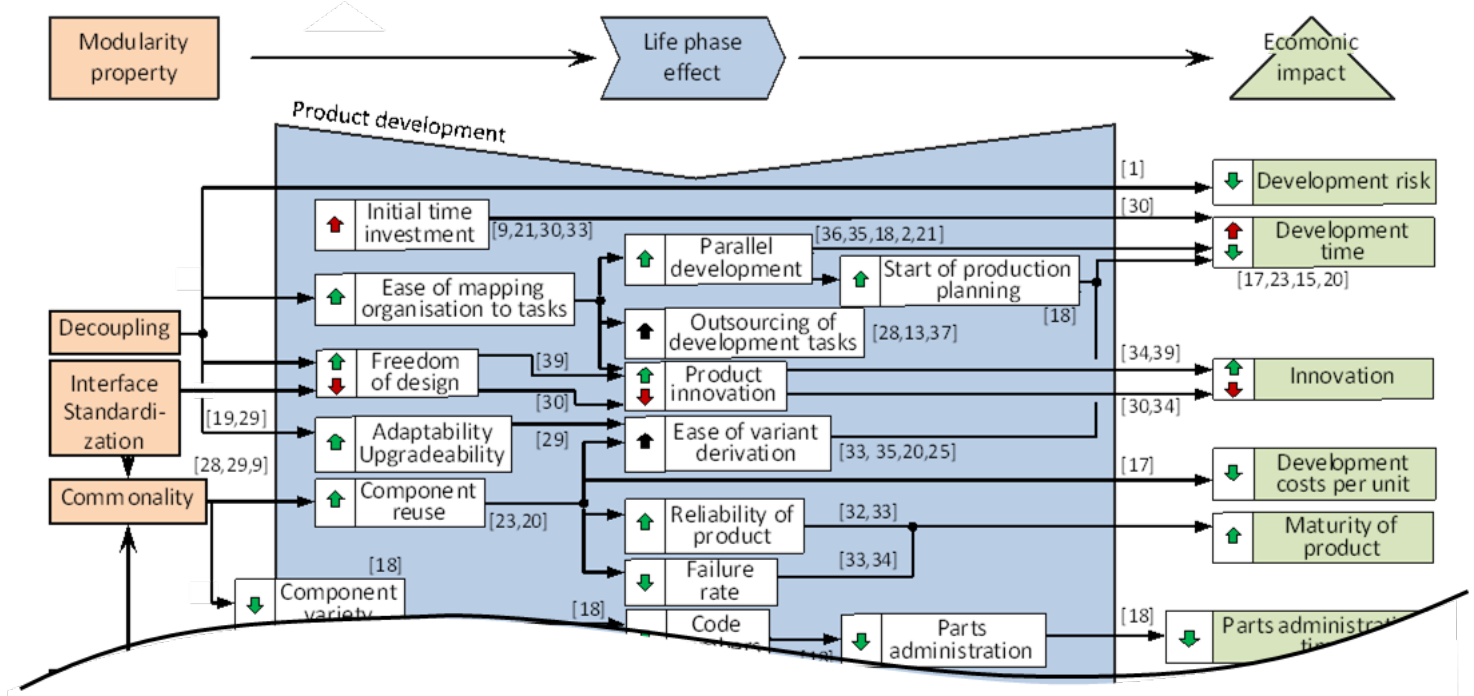

Bild 1: Ausschnitt des Wirkmodells modularer Produktstrukturen [2]

Das literaturbasierte Wirkmodell modularer Produktstrukturen beschreibt ausgehend von den Modularisierungseigenschaften und -merkmalen (Bild 1, links, orange) die Auswirkungen modularer Produktstrukturen in Form von Effektketten. Die Effektketten sind aus Primäreffekten und deren Folgeeffekten aufgebaut, wobei die Primäreffekte direkt aus den Modularisierungseigenschaften und -merkmalen resultieren. In dem Wirkmodell werden die möglichen Auswirkungen auf die Lebensphasen Produktentwicklung, Beschaffung, Produktion, Sales \& Marketing und Use \& Customer Service aufgezeigt [12]. Die Effektketten resultieren in den wirtschaftlichen Zielgrößen Zeit, Kosten, Qualität und Flexibilität, welche positiv oder negativ beeinflusst werden können (Bild 1, rechts, grün).

\subsection{Gestaltung von Produktionssystemen}

Ein Produktionssystem ist ein System, in dem Werte geschaffen werden. Produktionssysteme bestehen aus Teilsystemen. Das kleinste arbeitsfähige Element heißt Arbeitssystem. Die wesentliche Klassifizierung erfolgt nach Kriterien dem Fertigungsprinzip und gliedert die Produktion in Werkstattproduktion, Reihenproduktion oder Fließproduktionslinie. Die Form des Materialflusses und die Kontinuität des Materialflusses gelten als weitere Kriterien [13]. Für die Untersuchung von Produktionssystemen sind die logistischen Zielgrößen wie Durchlaufzeit, Leistung, Bestand und Reichweite von hoher Bedeutung [14][15]. Durch logistische Kennlinien lassen sich deren Abhängigkeiten aufzeigen. Logistische Kennlinien beschreiben die Mittelwerte der logistischen Zielgrößen Leistung, Durchlaufzeit, Reichweite und Übergangszeit in Abhängigkeit vom mittleren Bestand [16].

\subsection{Produktdifferenzierung versus Produktstandardisierung: Forschungsbedarf}

Trotz der gegenseitigen Einflüsse haben sich die Produktentwicklung und die Produktionsgestaltung weitestgehend separat entwickelt. In der Produktentwicklung und in der Produktionssystemgestaltung werden zudem gegensätzliche Ziele verfolgt. Standardisierte Produkte begünstigen die Gestaltung effizienter Produktionssysteme. So erfordert eine Fließbandmontage in der Regel eine hochgradige Produktstandardisierung. Dies steht im Widerspruch zur Strategie der Produktdifferenzierung in der Produktentwicklung, die einer vom Markt geforderten Variantenvielfalt gerecht wird [17]. Dieser Zielkonflikt wird unter anderem auch in dem Begriffspaar der Economies of Scale und Economies of Scope nach Porter [18] aufgegriffen. Porter befasst sich mit der Gegenüberstellung von Economies of Scale und 
Economies of Scope [18]. Economies of Scale beschreiben die Strategie der Kostenführerschaft. Dabei sollen möglichst niedrige Kosten der Leistungserbringung erreicht werden, um hohe Stückzahlen absetzen zu können. Die Strategie der Differenzierung wird durch die Economies of Scope beschrieben. Die Abgrenzung der angebotenen Leistungen muss erfolgen, um höhere Preise erzielen zu können. Die Differenzierung geschieht durch die Erfüllung individueller Kundenbedürfnisse. Der Zusammenhang zwischen den Strategien besteht darin, dass durch einen erhöhten Kundenfit höhere Preise erzielt werden, welche die negativen Effekte der Variantenvielfalt ausgleichen können.

Neben diesem Zielkonflikt stellen auch ein unterschiedliches Verständnis und eine unterschiedliche Wissensbasis der Fachdisziplinen eine Herausforderung dar, die Produktarchitekturgestaltung und der Produktionsgestaltung aufeinander abzustimmen. Wechselseitige Beeinflussungen werden bisher nicht durchgängig abgebildet, wodurch Potentiale in den jeweiligen Disziplinen, welche sich durch eine abgestimmte Entwicklung von Produktarchitektur und Produktionssystem ergeben können, nicht ausgeschöpft werden. Daraus ergibt sich die folgende Forschungsfrage:

Wie kann der Zusammenhang von der Produktarchitekturgestaltung und der Produktionssystemgestaltung durchgängig dargestellt und genutzt werden?

\section{Forschungsvorgehen}

Eine Antwort auf die Forschungsfrage stellt der MBSE-Ansatz dar, welcher nachfolgend vorgestellt wird. In der Produktentwicklung wird dieser Ansatz zur Beherrschung der Komplexität bereits als vielversprechend angesehen [19] [20]. Als Datenbasis werden zentrale Systemmodelle in spezialisierten Softwaretools aufgesetzt. Dies ermöglicht es, Datenzusammenhänge in unterschiedlichen Ansichten und interaktiven Diagrammen darzustellen [20].

Das durchgängige Datenmodell setzt auf einem bereits existierenden SysML-Modell des Wirkmodells modularer Produktstrukturen auf [21]. Es erfolgt eine Erweiterung des Modells um Modultreiber (siehe Absatz 4.2). Dafür wird zunächst eine Literaturrecherche zu existierenden Modultreibern durchgeführt. Das von Erixon etablierte Konzept [5] wurde in vielen weiteren Modularisierungsmethoden und auch Methodenanpassungen aufgegriffen. Im Rahmen dieses Papers wurde die von Blees durchgeführte Literaturrecherche (7 Literaturquellen, inklusive des angepassten Modultreiberkonzepts nach Blees) zu produktstrategischen Modularisierungsmethoden [6] um eine darauf aufbauende Literaturrecherche erweitert. Für die erweiterte Literaturrecherche wurde zunächst die Datenbank Scopus durchsucht. Folgende Suchbegriffe wurden für die Suche in Titel, Abstracts und Keywords verwendet: („module driver*“ $)=75$ Suchergebnisse, („driver of modular*“) $=1$ Suchergebnis und ((,,strategic drivers“) AND (modular*) $)=8$ Suchergebnisse. Die Ausdehnung des Datenbankenumfangs auf die Design Society und Web of Science ergab keine weiteren, von Scopus nicht abgedeckten, Treffer. Nach dem Ausschluss von Duplikaten und der anschließenden Sichtung der Suchergebnisse wurden 9 Suchergebnisse als relevant eingestuft, und die anfängliche Literaturrecherche um diese erweitert. Bei dem Abgleich der unterschiedlichen Suchergebnisse wird klar, dass die Modultreiber nach Erixon vermehrt aufgegriffen oder angepasst werden. Ein Beispiel dafür stellt der Modultreiber Verfügbarkeit von Lieferanten dar [22], welcher einen Zusammenhang zu Erixons Modultreiber BlackboxZukauf aufweist.

Die Modultreiber werden in einem nächsten Schritt den Produktlebensphasen zugeordnet und anschließend mit den Elementen des Wirkmodells modularer Produktstrukturen, also den Eigenschaften und Merkmalen der Modularität und den Effekten, abgeglichen. Ein Beispiel für einen bekannten Modultreiber aus der Produktion ist das separate Testen. Der Modultreiber separates Testen ist zudem als Effekt, welcher durch die Kombinierbarkeit auftritt, in dem Wirkmodell modularer Produktstrukturen in der Lebensphase Produktion zu finden [12]. Ein 
weiteres Beispiel für einen Modultreiber der Produktion ist die Gleichteilstrategie, welche einen Zusammenhang zu der Modularisierungseigenschaft Kommunale Verwendung aufweist.

Das Analyseergebnis stellt das verknüpfende Element zwischen den Modultreibern von produktstrategischen Modularisierungsmethoden und dem Wirkmodell modularer Produktstrukturen dar (Bild 2).

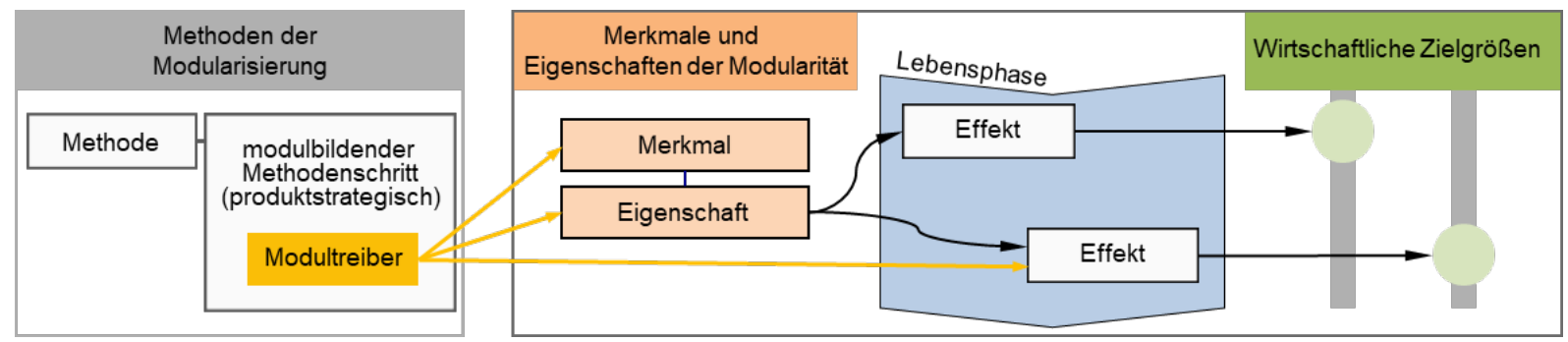

Bild 2: Mögliche Anknüpfungen von Modultreibern an das Wirkmodell modularer Produktstrukturen

In dem Bild sind die möglichen Anknüpfungspunkte von Modultreibern produktstrategischer Modularisierungsmethoden an das Wirkmodell modularer Produktstrukturen schematisch dargestellt. Demnach ist der jeweilige Anknüpfungpunkt modultreiberspezifisch.

Als weiteres Element soll der produktionssystemabhängige Zusammenhang zwischen den wirtschaftlichen Effekten in der Produktion und der internen Vielfalt mithilfe einer qualitativen Kennlinie abgebildet werden und als Zusatzinformation in das Datenmodell integriert werden können (siehe Abschnitt 4.3).

\section{Modellierung der Datenzusammenhänge in SysML}

In Kapitel 4 werden aufbauend auf der Modellierung der Datenzusammenhänge die Modultreiber an das Wirkmodell exemplarisch angeknüpft. Weiterhin werden logistische Kennlinien im Modell hinterlegt, sodass die Analyse der Datenzusammenhänge ermöglicht wird.

\subsection{Vorgehen zur Modellierung von Datenzusammenhängen in SysML}

Um ein durchgängiges Datenmodell zur Darstellung der Zusammenhänge zwischen der Produktentwicklung und der Produktionssystemgestaltung initial aufzusetzen, wird zunächst die Systemgrenze definiert. Anschließend erfolgt die Analyse der einzelnen Systemelemente hinsichtlich ihrer Abhängigkeiten. In einem daraus abgeleiteten Metadatenmodell werden diese Informationen gesammelt. Ein Metadatenmodell stellt dabei ein Modell dar, das die Modellierung beschreibt [23, 24] und eine konsistente Datengrundlage ermöglicht. Es stellt den Grundstein der Modellierung der Datenzusammenhänge in SysML dar.

Für die Modellierung wird dabei der Cameo Systems Modeler verwendet. Die Modellierungssoftware ermöglicht die Erstellung von Diagrammen und Tabellen, die auf der SysML-Notation aufbauen [20]. Einzelne Systemelemente und ihre Verknüpfungen sind jeweils nur einmal in dem Modell enthalten. Mithilfe der unterschiedlichen Diagramme und Tabellen können einzelne Ansichten des Datenmodells generiert werden.

\subsection{Anknüpfung von Modultreibern an das Wirkmodell modularer Produktstrukturen}

Die Modellinhalte werden so dokumentiert, dass das Modell von Vertretern beider Disziplinen verwendbar ist und mit weiteren fachspezifischen Inhalten gefüllt werden kann. Ziel ist es, so einen Grundstein für ein abgestimmtes und konsistentes Datenmodell zu legen.

Die Darstellung der Datenzusammenhänge des Wirkmodells modularer Produktstrukturen erfolgt in einem Blockdefinitionsdiagramm (Bild 3, angelehnt an [21]). 


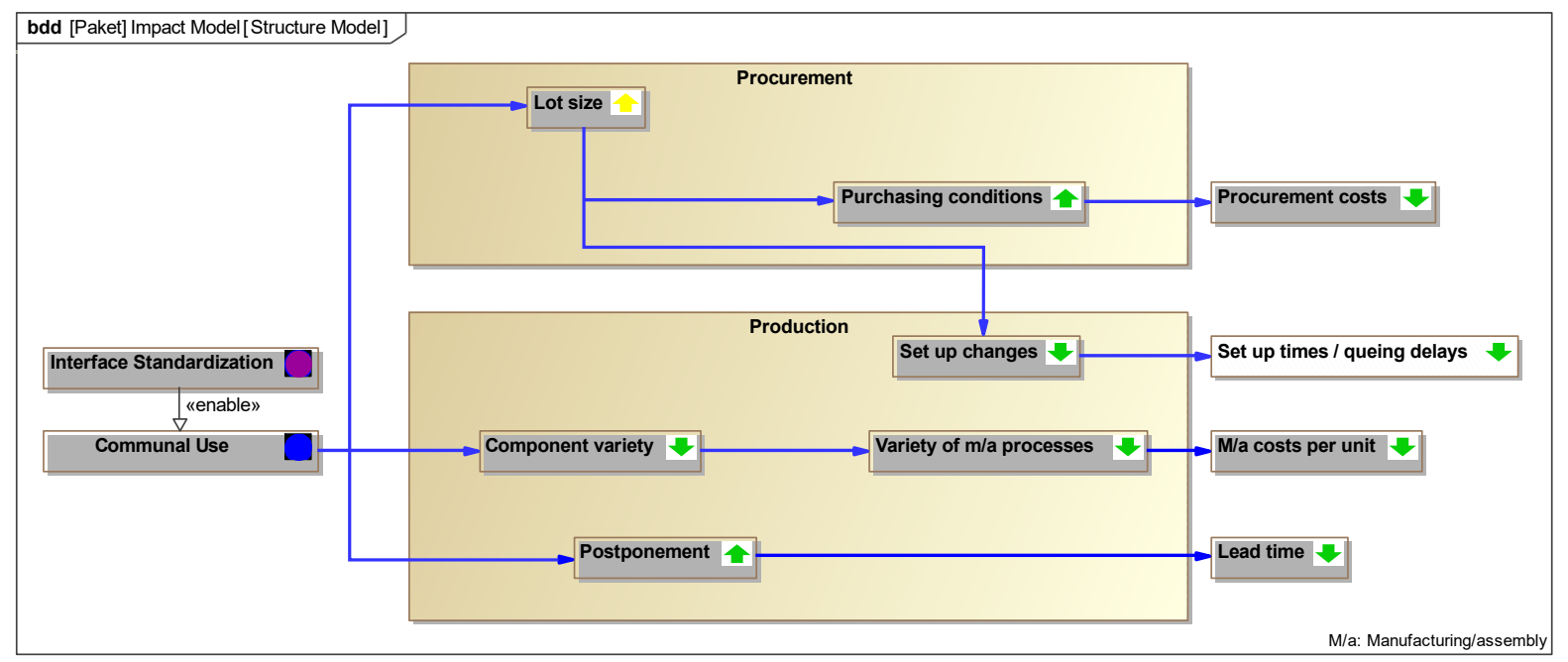

Bild 3: Ausschnitt des Wirkmodells modularer Produktstrukturen in SysML (angelehnt an [21])

Die einzelnen Elemente sind Blöcke, welche mit Abhängigkeiten untereinander verbunden sind. Durch die Verwendung von Stereotypen können die Blöcke in Eigenschaften und Merkmale der Modularität, Lebensphasen und Effekte unterschieden werden. Die Anordnung orientiert sich dabei an der Visualisierung des Wirkmodells modularer Produktstrukturen (vgl. [2], Bild 1). Durch die Verwendung von Icons lassen sich den Eigenschaften und Merkmalen Farben zuordnen. Auch die Ausprägung der einzelnen Effekte lässt sich in Form von Icons hinterlegen.

Das Ergebnis der zuvor durchgeführten Literaturrecherche zu den Modultreibern wird anschließend in SysML integriert. Die Modultreiber werden dafür als Blöcke dargestellt, welche durch den stereotyp $<<$ moduledriver $>>$ als solche gekennzeichnet werden. Die Erstellung der Abhängigkeiten der Modultreiber durch den Verknüpfungstyp <<allocate>> zu den Elementen des Wirkmodells modularer Produktstrukturen erfolgt separat in einem weiteren Blockdefinitionsdiagramm. Ein Ausschnitt des Blockdefinitionsdiagramms ist in Bild 4 dargestellt.

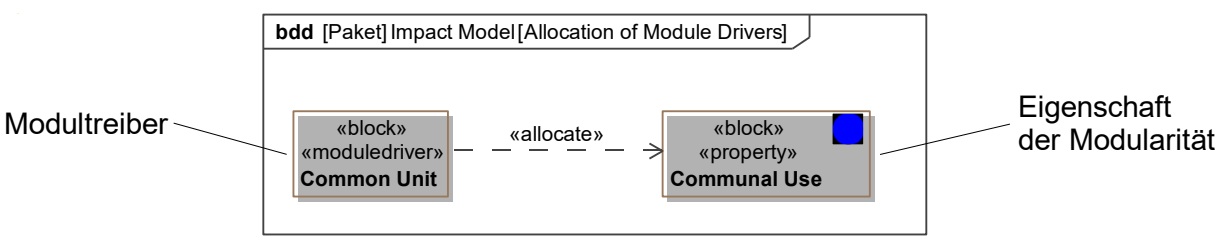

Bild 4: Anknüpfung von Modultreibern und dem Wirkmodell

Der Modultreiber Gleichteilstrategie (Common Unit) wird der Modularisierungseigenschaft Kommunale Verwendung (Communal Use) zugeordnet. Bei dem Block Communal Use handelt es sich dabei um dasselbe Element wie in Bild 3. Durch die separate Verknüpfung der Modultreiber mit den Elementen des Wirkmodells bleibt die Übersichtlichkeit (vgl. Bild 3) erhalten.

\subsection{Konzept zur Anknüpfung von Informationen zur Produktionssystemgestaltung}

Für wesentliche Produktionssystemausprägungen sollen im Modell Kennlinien hinterlegt werden, die die logistischen Zielgrößen in Abhängigkeit von der internen Vielfalt darstellen. Dafür sollen die bestehenden Modelle so erweitert werden, dass sie die Auswirkung der internen Vielfalt abbilden können. Danach werden die Abhängigkeiten der logistischen 
Zielgrößen im Modell als Zusatzinformation hinterlegt. Bild 5 zeigt ein qualitatives Beispiel für diese Kennlinien.

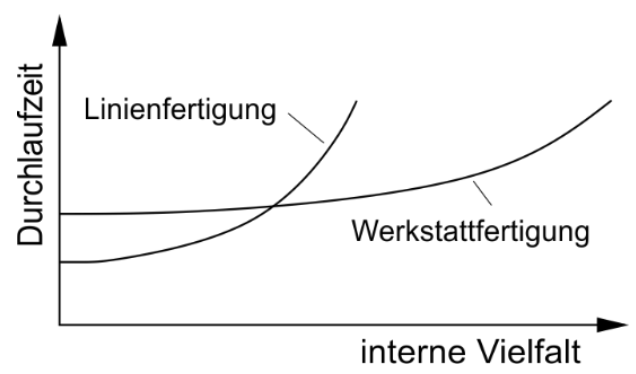

Bild 3: Logistische Zielgrößen verschiedener Produktionssystemausprägungen

Die Durchlaufzeit von Aufträgen steigt demnach mit zunehmender interner Vielfalt des Produktes. Für verschiedene Produktionssystemausprägungen (z.B. Linienfertigung oder Werkstattfertigung) unterscheiden sich diese Verläufe erheblich. Die Linienfertigung ermöglicht bei einer geringen internen Vielfalt sehr kurze Durchlaufzeiten. Mit steigender interner Vielfalt nimmt die Durchlaufzeit jedoch stark zu. Im Vergleich dazu resultiert eine Werkstattfertigung auch bei geringer Vielfalt nicht in ebenso kurzen Durchlaufzeiten. Die Durchlaufzeit in der Werkstattfertigung ist jedoch deutlich robuster gegenüber einer steigenden internen Vielfalt. Diese Modellierung bildet die Basis, um die Produktionssystemgestaltung in der Modellierung zu berücksichtigen.

\subsection{Analyse der Datenzusammenhänge}

Mithilfe ausgewählter Diagrammarten des Cameo Systems Modelers lassen sich die zuvor beschriebenen Datenzusammenhänge des SysML-Modells analysieren. Durch die Verwendung der Relation Map können indirekte Zusammenhänge zwischen einzelnen Modellelementen aufgezeigt werden (Bild 6).
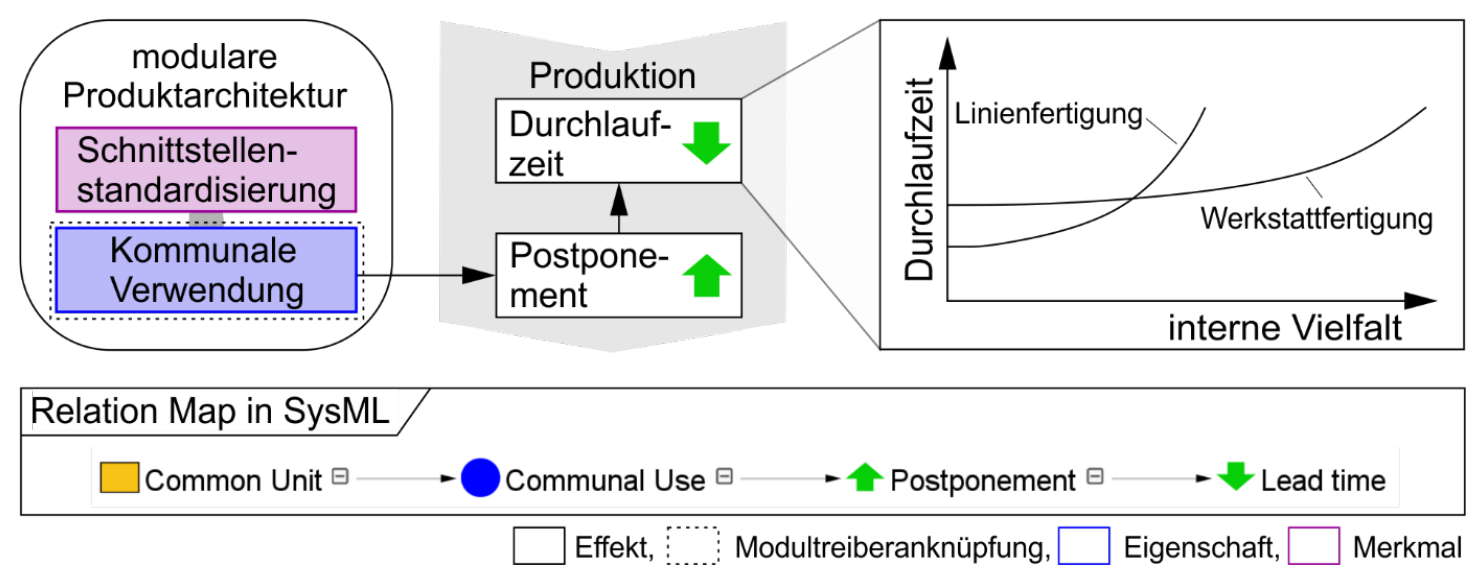

Bild 6: Durchgängige Datenmodellierung in SysML

Bei der Analyse der Zusammenhänge in SysML wird ersichtlich, dass durch die Anknüpfung des Modultreibers an das Wirkmodell modularer Produktstrukturen ein indirekter Zusammenhang zwischen diesem Modultreiber und der Durchlaufzeit besteht.

Durch die Modularisierung von Produktstrukturen verringert sich die interne Vielfalt bei gleichbleibender externer Vielfalt. Wird nun zusätzlich der Modultreiber Gleichteilstrategie während der Durchführung einer Modularisierungsmethode angewandt, kann dies dazu führen, dass diese Auswirkung verstärkt wird, was einen möglichen Wechsel des Produktionssystems begünstigt. 


\section{Diskussion und Ausblick}

In Kapitel 4 wurde durch die Darstellung des Modultreibers Gleichteilstrategie ein Anschauungsbespiel gewählt, in dem der Modultreiber einer Modularisierungseigenschaft zugeordnet werden kann. Bei der Literaturrecherche zu Modultreibern und dem anschließenden Abgleich mit den Elementen des Wirkmodells zeigte sich jedoch, dass einige Modultreiber auch mit Effekten des Wirkmodell zusammenhängen, wie beispielsweise das separate Testen. Teilweise ist auch keine Zuordnung jeglicher Art möglich.

In weiterführender Forschung sollen die Abhängigkeitsarten aus Bild 2 weiter kategorisiert und zudem ein Konzept entwickelt werden, um Modultreiber, die derzeit nicht im Zusammenhang mit dem Wirkmodell modularer Produktstrukturen stehen, in dieses zu integrieren.

In Kapitel 4 wurde aufgezeigt, dass die Wahl bzw. Fokussierung von Modultreibern bei der Anwendung von Modularisierungsmethoden über das Wirkmodell modularer Produktstrukturen in den Zusammenhang mit der Auswahl von Produktionssystemen gebracht werden kann. Durch die schrittweise Modellierung der einzelnen Zusammenhänge in SysML konnten die Datenzusammenhänge zurückverfolgbar aneinandergekoppelt werden. Die Modellierung der Zusammenhänge in SysML bietet dabei den großen Vorteil, dass die indirekten Verknüpfungen durch die direkten Kopplungen konsistent aufgezeigt werden können. Zudem stellt das SysML-Modell eine Grundlage für eine interdisziplinäre Nutzung dieser Informationen dar. Durch die offen einsehbare Logik hinter der Modellierung im MBSE-Kontext lassen sich die bisher erstellten Zusammenhänge konsistent um weitere Inhalte gegenwärtiger Forschung erweitern.

Die Schnittstellen zwischen der Produktarchitekturgestaltung und der Produktionssystemgestaltung sind jedoch weitaus vielfältiger, sodass eine eingehende interdisziplinäre Betrachtung aus den beiden Bereichen Produktentwicklung und Produktionsmanagement große Potenziale birgt. Das initiale SysML-Modell soll daher in weiterer interdisziplinärer Forschung zwischen der Produktentwicklung und dem Produktionsmanagement in einem nächsten Schritt um Kennlinien erweitert werden, um ausgehend von der Produktion eine fundierte Grundlage für die Unterstützung der Entscheidungen in der Modulbildung zu liefern. Der Nutzen des Ansatzes soll dabei durch eine Expertenevaluation bestätigt werden.

Des Weiteren sollen neben der Implementierung von Modultreibern in das Datenmodell ebenfalls die einzelnen Schritte von unterschiedlichen Modularisierungsmethoden aufgenommen werden. Das geplante Vorgehen dazu orientiert sich an dem Vorgehen zur Modellierung ausgewählter Elemente des integrierten PKT-Ansatzes zur Entwicklung modularer Produktfamilien als Prozessmodell in Aktivtätsdiagrammen nach Hanna [25]. Das Ziel ist dabei, frühzeitig mögliche Auswirkungen zu erkennen, die durch die Durchführung unterschiedlicher Modularisierungsmethoden erfolgen können. Somit kann die Wahl einer Modularisierungsmethode hinsichtlich ihrer Auswirkungen an die gegebenen Umstände in Modularisierungsprojekten angepasst und dadurch vereinfacht werden. Zudem können die in der Literatur bestehenden Modularisierungsmethoden miteinander verglichen werden und eine gezielte Anpassung von Methodenschritten einzelner Methoden für die Erweiterung wird unterstützt.

\section{Danksagung}

Die dargestellten Ergebnisse wurden im Rahmen des Forschungsprojektes „WiMo 2 Entwicklung eines Wirkmodells der Eigenschaften modularer Produktstrukturen zur Bewertung methodischer Ansätze“ [Projektnummer: 276691857] entwickelt, das von der Deutschen Forschungsgemeinschaft (DFG) gefördert wird. 


\section{Literaturverzeichnis}

[1] Krause, Dieter; Gebhardt, Nicolas: Methodische Entwicklung modularer Produktfamilien: Hohe Produktvielfalt beherrschbar entwickeln. Berlin: Springer Vieweg, 2018. https://doi.org/10.1007/978-3-662-53040-5

[2] Hackl, Jennifer et al.: Impact of Modularity Decisions on a Firm's Economic Objectives. In: Journal of Mechanical Design 142, Issue 4 (2020): Nr. 041403. https://doi.org/10.1115/1.4044914

[3] Krause, Dieter and Ripperda, Sebastian: An Assessment of methodical Approaches to support the Development of Modular Product Families. In: Proceedings of the 19th International Conference on Engineering Design (2013), S. 31-40.

[4] Stone, Robert B.: Towards a Theory of modular Design. Dissertation. The University of Texas at Austin, 1997.

[5] Erixon, Gunnar: Modular Function Deployment - A Method for Product Modularisation. Dissertation. The Royal Institute of Technology. Sweden, 1998.

[6] Blees, Christoph: Eine Methode zur Entwicklung modularer Produktfamilien. Dissertation. Technische Universität Hamburg-Harburg. Hamburg, 2011.

[7] Windheim, Marc: Cooperative Decision-Making in Modular Product Family Design. Dissertation. Technische Universität Hamburg. Hamburg, 2020. https://doi.org/10.1007/978-3-662-60715-2

[8] Harland, Peter E. and Uddin, Zakir: Effects of product platform development: fostering lean product development and production. In: International Journal of Product Development, Vol. 19 (2014), S. 259-285. https://doi.org/10.1504/IJPD.2014.064881

[9] Fixson, Sebastian K.: A Roadmap for Product Architecture Costing. In: Product platform and product family design methods and applications, Simpson, Timpthy W. et al. New York: Springer US, 2006, S. 305-334. https://doi.org/10.1007/0-387-29197-0_13

[10] Antonio, K.W. Lau; Yam, Richard C.M.; Tang, Esther: The impacts of product modularity on competitive capabilities and performance: An empirical study. In: International Journal of Production Economics, Vol. 105 (2007), S. 1-20. https://doi.org/10.1016/j.ijpe.2006.02.002

[11] Hackl, Jennifer.; Krause, Dieter: Effects of modular product structures on life phases and economic factors. In: Proceedings of the DESIGN 2016 14th International Design Conference (2016), S.1285-1294.

[12] Hackl, Jennifer.; Krause, Dieter: Towards an impact model of modular product structures. In: Proceedings of the 21st International Conference on Engineering Design (2017), S.151-160.

[13] Wiendahl, Hans-Peter: Betriebsorganisation für Ingenieure. Carl Hanser Verlag GmbH Co KG, 2014. https://doi.org/10.3139/9783446441019

[14] Lödding, Hermann, Verfahren der Fertigungssteuerung: Grundlagen, Beschreibung, Konfiguration. VDI-Buch, 3. Auflage, Springer Vieweg, Berlin, Heidelberg, 2016. https://doi.org/10.1007/978-3-662-48459-3_29

[15] Wiendahl, Hans-Peter: Fertigungsregelung: Logistische Beherrschung von Fertigungsabläufen auf Basis des Trichtermodells. München: Hanser, 1997.

[16] Nyhuis, Peter; Wiendahl, Hans-Peter: Logistische Kennlinien: Grundlagen, Werkzeuge und Anwendungen. VDI-Buch, 3. Aufl. 2012. Springer: Berlin, Heidelberg, 2012. https://doi.org/10.1007/978-3-540-92839-3

[17] Bohl, Arne: Kennlinien der Produkt- und Produktionskomplexität. Dissertation. Rheinisch-Westfälische Technische Hochschule Aachen, Fakultät für Maschinenwesen, 2014.

[18] Porter, Michael E.: Competitive advantage: Creating and sustaining superior performance, 1. Free Press export ed.. Free Press: New York, 2004.

[19] Bursac, Nikola; Albers, Albert; Schmitt, Thomas: Model based systems engineering in modular design-a potential analysis using portal type scraper reclaimers as an example. In: Procedia CIRP, 50 (2016), S. 802807. https://doi.org/10.1016/j.procir.2016.04.156

[20] Weilkiens, Tim: Systems Engineering mit SysML/UML: Anforderungen, Analyse, Architektur. 3. Überarbeitete und aktualisierte Auflage. Heidelberg: dpunkt Verlag, 2014.

[21] Schwede, Lea-Nadine. et al.: Consistent Modelling of the Impact Model of Modular Product Structures with Linking Boundary Conditions in SysML. In: Proceedings of the 22nd ICED (2019), S. 3601- 3610. https://doi.org/10.1017/dsi.2019.367

[22] Lange, Mark W.; Imsdahl, Andrea: Modular Function Deployment: Using Module Drivers to Impart Strategies to a Product Architecture. In: Simpson, Timothy W.; Jiao, Jianxin; Siddique, Zahed; Hölttä-Otto, Katja (eds): Advances in Product Family and Product Platform Design. Springer, New York, 2014.

[23] Holt, Jon: Model-based requirements engineering for system of systems. In 2012 7th International Conference on System of Systems Engineering (SoSE) (2012), S. 561-566. https://doi.org/10.1109/SYSoSE.2012.6384145

[24] Stachowiak, Herbert: Allgemeine Modelltheorie. Wien: Springer,1973. https://doi.org/10.1007/978-3-70918327-4

[25] Hanna, Michael; Schwenke, Johann; Krause, Dieter: Inconsisteny Management for Product Families with many Variants through a Model-based Approach in Modular Lightweight Design. In: Proceedings of the Design Society: DESIGN Conference (2020), S. 917-926. https://doi.org/10.1017/dsd.2020.309 